\title{
Tanshinone IIA elevates serum soluble klotho levels and decreases cardiovascular events in patients on maintenance hemodialysis: a prospective before-after study
}

\author{
Qin Xu", Haitao Li", Xu Zhang, Hao Ding, Juan Cao \\ Department of Nephrology, Taixing People's Hospital, Taixing, China \\ Contributions: (I) Conception and design: Q Xu, H Li, J Cao; (II) Administrative support: J Cao; (III) Provision of study materials or patients: Q \\ Xu, H Li; (IV) Collection and assembly of data: Q Xu, H Li, X Zhang, H Ding; (V) Data analysis and interpretation: Q Xu, H Li; (VI) Manuscript \\ writing: All authors; (VII) Final approval of manuscript: All authors. \\ "These authors contributed equally to this work. \\ Correspondence to: Juan Cao, MD. Department of Nephrology, Taixing People's Hospital, 1 Changzheng Road, Taixing, China. \\ Email: caojuan20191@163.com.
}

Background: Cardiovascular disease (CVD) is the most common adverse event of maintenance hemodialysis (MHD), in which serum soluble klotho plays a vital role. Tanshinone IIA (tan) has been used to protect the cardiovascular system for hundreds of years. This study was performed to investigate the effects of tan on klotho level and cardiovascular events of MHD patients.

Methods: Totally 112 patients were enrolled in this study, and their demographic, clinical, and laboratory characteristics were collected at admission. Serum soluble klotho, carotid intima-media thickness (CIMT), and abdominal aorta calcification (AAC) level were measured to determine their relationship. Seventy-one patients were given sodium tan sulfonate injection according to their willingness, while 41 patients were not given any specific treatment. The endpoint events were recorded, including cardiovascular mortality, nonfatal cardiovascular events, and all-cause mortality.

Results: All patients were divided into two groups on whether the level of serum soluble klotho was more than medium or not. CIMT and AAC grade showed significant differences between 2 groups (group of low level of klotho versus group of high level of klotho, CIMT: $1.03 \pm 0.22$ vs. $0.85 \pm 0.20 \mathrm{~mm}, \mathrm{P}<0.001$; AAC grade: $5.04 \pm 3.93$ vs. $1.69 \pm 2.30$ points, $\mathrm{P}<0.001)$. All patients were followed up for at least one year. The results revealed that using tan improved the level of serum soluble klotho $(490.23 \pm 153.97 \mathrm{pg} / \mathrm{mL}$ after using tan versus $444.49 \pm 143.32 \mathrm{pg} / \mathrm{mL}$ before using tan, $\mathrm{P}=0.042)$. Kaplan-Meier curves showed that cardiovascular event-free survival was significantly higher in patients given $\tan (\mathrm{P}=0.040)$ compared with patients not given tan.

Conclusions: Tan effectively increases the level of serum soluble klotho and further reduces the incidence of cardiovascular events in MHD patients.

Keywords: Tanshinone IIA; klotho; cardiovascular events; maintenance hemodialysis (MHD)

Submitted May 12, 2020. Accepted for publication Jul 08, 2020.

doi: 10.21037/apm-20-1172

View this article at: http://dx.doi.org/10.21037/apm-20-1172 


\section{Introduction}

Along with the development of living conditions and the aging population, the incidence of chronic kidney disease $(\mathrm{CKD})$ and subsequent end-stage renal disease (ESRD) is rapidly increasing, and they have raised more and more attention around the world nowadays. The incidence of CKD in China is reported to be $10.8 \%$, and ESRD patients are estimated to be 1 million $(1,2)$. Once the development of end-stage renal disease takes place, the most effective treatment is a renal replacement. Nevertheless, the scarcity of kidney donors and the prohibitive cost cause most patients to be unable to receive kidney replacement therapy. Alternatively, hemodialysis and peritoneal dialysis have become the most important therapy for ESRD patients.

At present, there are 700,000 people undergoing maintenance hemodialysis (MHD) in China (3). The concept of hemodialysis was firstly proposed in 1840s and the first patient received hemodialysis in 1926, which failed to work. Afterwards, Willem Kolff innovatively improved the device of hemodialysis and cured the first patient with acute kidney injury (4). After decades of improvement, hemodialysis has become very mature. However, several adverse events may occur during MHD treatment. Cardiovascular disease (CVD) is the most common adverse event of ESRD patients undergoing MHD, including coronary artery disease, congestive heart failure, stroke, and peripheral arteriosclerotic disease (5). As reported, over half of ESRD patients died of CVD, and most CKD patients have clear cardiovascular system dysfunction before ESRD (6). Cardiovascular system dysfunction, the early manifestation of CVD, includes arteriosclerosis, endothelial dysfunction, the increase of carotid intimamedia thickness (CIMT), and vascular calcification. It has been revealed that vascular dysfunction is one of the main causes of cardiovascular death in ESRD patients and an independent predictor of CVD $(7,8)$. Thus, there is an urgent requirement to understand the mechanism of CVD during MHD and find efficient therapy to prevent or even reverse the development of CVD.

In recent years, serum soluble klotho protein has been reported to be associated with CVD. Klotho, a $130 \mathrm{kDa}$ transmembrane protein, is expressed in the distal convoluted tubules of the kidneys as well as some other organs (9). It is originally found to be involved in human aging and the regulation of calcium and phosphorus metabolism $(10,11)$. The latest studies have demonstrated klotho also participants in anti-oxidation, anti-apoptosis, promotion of angiogenesis, inhibition of fibrosis, and protection of stem cells (12). Memmos et al. found that a low level of soluble klotho is associated with cardiovascular mortality and adverse events in MHD patients (13). Also, a low level of soluble klotho increases the coronary artery calcification and CIMT $(14,15)$. It may be feasible to develop novel therapy of CVD with it, considering the vital role of serum soluble klotho in CVD among MHD patients.

Tanshinone IIA ( $\tan )$, an active ingredient isolated from the rhizomes of the Chinese herb Salvia miltiorrhiza, has been used to treat CVDs for hundreds of years (16). Its effects of protecting the cardiovascular system are mainly on its abilities of vasodilator (17), anti-inflammation (18), and scavenging free radical (19). However, it remains to be explored whether tan is involved in the regulation of serum soluble klotho levels and the improvement of cardiovascular dysfunction in MHD patients.

Here, in the present study, we performed a prospective before-after intervention study to verify the role of serum soluble klotho in MHD patients, to investigate further the effects of tan on cardiovascular events in MHD patients. We present the following article in accordance with the STROBE reporting checklist (available at http://dx.doi. org/10.21037/apm-20-1172).

\section{Methods}

\section{Setting and study design}

Our study was a prospective before-after study of MHD patients admitted to Taixing People's Hospital between January 2018 and December 2018. All adult patients, who signed the informed consent, were older than 18 years, underwent MHD thrice a week for more than three months and were in stable condition, were enrolled in this study. The following patients were excluded from this study: patients with (I) serious CVD, (II) malignant disease, (III) acute infectious diseases, (IV) severe malnutrition, (V) trauma, and (VI) recent surgery. The study was performed in compliance with the ethical principles of the Declaration of Helsinki (as revised in 2013), and had been approved by the ethics committee of Taixing People's Hospital.

\section{Baseline assessment}

Baseline data of enrolled patients were recorded when they were admitted to the hospital for the first time. The recorded data included demographic data, blood pressure, 
comorbid diseases, history of smoking, dialysis duration, serum soluble klotho level, CIMT, abdominal aorta calcification (AAC) level, and some biochemical indexes including hemoglobin, albumin, cholesterol, creatinine, C-reactive protein, calcium, and phosphorus. Skilled operators performed the above measurements, and blood samples were collected at the first admission of enrolled patients.

The levels of serum soluble klotho were measured using an enzyme-linked immunosorbent assay (ELISA) system (Immuno-Biological Laboratories Co., Ltd, Tokyo, Japan) according to the manufacturer's instructions. Briefly, add $100 \mu \mathrm{L}$ loading buffer, standard solution or sample solution to the blank wells, standard wells or sample wells to be tested in the ELISA plate respectively. The ELISA plate was then incubated at $37^{\circ} \mathrm{C}$ for $90 \mathrm{~min}$. Then discarded the solution, added $100 \mu \mathrm{L}$ detection working solution into each well, and incubated at $37^{\circ} \mathrm{C}$ for $1 \mathrm{~h}$. Wash the plate for three times, add $100 \mu \mathrm{L}$ HRP converge working solution to each well, and incubate at $37^{\circ} \mathrm{C}$ for $30 \mathrm{~min}$. Wash the plate for five times. Add $90 \mu \mathrm{L}$ substrate solution and incubate at $37{ }^{\circ} \mathrm{C}$ in dark for 15 minutes. The optical density of each well was measured at $450 \mathrm{~nm}$. The assay kit has a lower limit of detection of $6.15 \mathrm{pg} / \mathrm{mL}$, with intra-assay and interassay coefficients of variation less than $10 \%$.

CIMT was measured according to an earlier study (14). Briefly, ultrasonography of the carotid arteries was performed in each patient who underwent using a highresolution real-time scanner. Patients were in the supine position during the examination. The carotid artery was scanned in both the longitudinal and transverse directions. The distance between the lumen-intima interface and the media-adventitia interface was calculated, and the location of the atherosclerotic lesion showing the greatest distance was the maximum intima-media thickness (IMT) value. Additionally, as a special lesion in the blood vessels, a plaque was defined as localized thickening rather than a circumferential change. The greatest thickness of intimamedia lesions was found as the maximum IMT value.

AAC levels were also measured according to an earlier study (20). Lateral plain radiography of the abdomen was performed, which ranged from the last two thoracic vertebrae to the first two sacral vertebrae. The tubular structure coursing in front of the anterior surface from the first to the fourth lumbar vertebra was showed as the abdominal aorta. AAC in front of each was analyzed separately for the posterior and anterior aorta wall. Calcification was divided into four grades. AAC levels were divided into four grades as follows: 0 points with no calcific deposits, 1 point with scattered calcific deposits filling less than one-third length of the corresponding lumbar vertebral level, 2 points with medium calcific deposits filling more than one-third, but less than the two-thirds length of the corresponding lumbar vertebral level; 3 points with many calcific deposits filling more than the two-thirds length of the corresponding lumbar vertebral level. For this grading system, the grade of AAC levels ranged from a minimum of 0 points to a maximum of 24 points.

\section{Interventions}

Some enrolled MHD patients in this study were given sodium tan sulfonate injection with a dosage of $80 \mathrm{mg} / \mathrm{q} . \mathrm{d}$. (once a day) according to their willingness. Other patients were not given any specific treatment. Cardiovascular parameters, including CIMT and AAC levels in enrolled patients, were measured each month. During the followup, the endpoints of patients in the two groups were compared to assess the efficiency of tan on the treatment of cardiovascular dysfunction in MHD patients.

\section{Endpoints}

All enrolled patients were followed up for at least one year. The endpoints in this study included cardiovascular mortality (patients died of fatal cardiovascular events occurring in 30 days, including myocardial infarct and stroke), non-fatal cardiovascular events (including myocardial infarct and stroke), and all-cause mortality.

\section{Statistical analysis}

SPSS (version 20.0) was used for all data analyses. Categorical variables in the study were reported as percentages, while continuous variables were reported as the means with standard deviations. All the continuous variables were analyzed with a homogeneity test, and then we decided to use 2-tailed $t$-tests or Mann-Whitney tests for comparisons between patients of the two groups on the result of the homogeneity test. However, the categorical variables are analyzed using $\chi^{2}$ tests. To explore the risk factors of cardiovascular dysfunction, univariate, and multivariate linear regression analysis was performed. Survival curves of patients were generated on the KaplanMeier method and compared using the log-rank test. The level of significance was set at the conventional level 


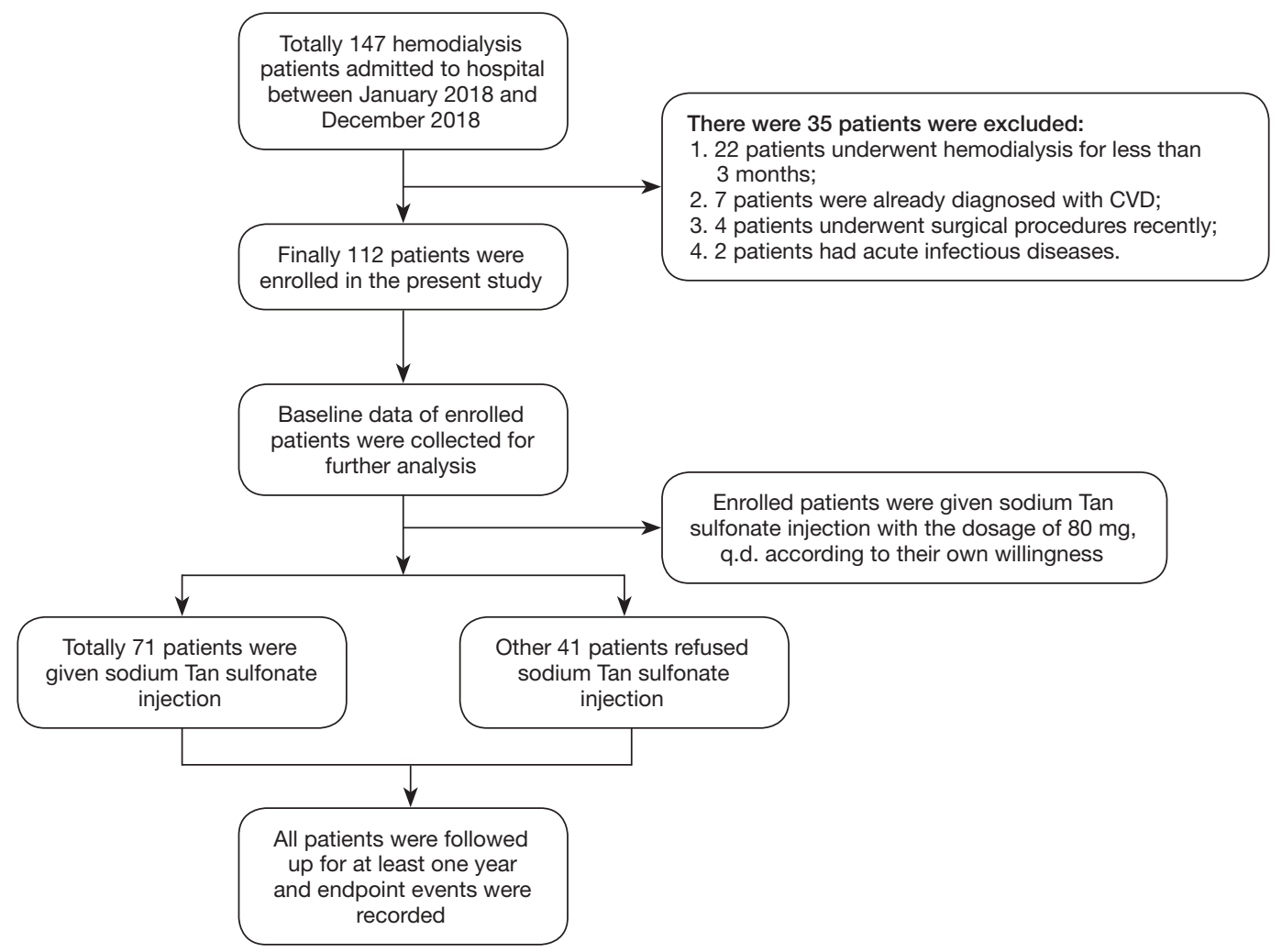

Figure 1 Flow chart of this study.

of $\alpha=0.05$. All $P$ values were two-sided, and $P<0.05$ was considered statistically significant.

\section{Results}

\section{Patient baseline characteristics}

Between January 2018 and December 2018, 147 patients were admitted to our hospital for MHD. Among the patients admitted, 35 patients were excluded following exclusion criteria, as shown in Figure 1. Finally, 112 patients were enrolled in this study. Their demographic, clinical, and laboratory characteristics were collected and listed in Table 1 . The median age of enrolled patients was $60.13 \pm 9.30$ years old, and 65 of all patients were male. Their median body mass index was $22.75 \pm 3.07$. Most enrolled patients had undergone MHD for several years, and the mean of dialysis duration was $63.09 \pm 29.54$ months. Hypertension and diabetes mellitus were the most common comorbid diseases among these patients. There were 84 (75\%) and $25(22.32 \%)$ patients diagnosed with hypertension and diabetes mellitus, respectively. Besides, 59 patients in all were smokers or used to be smokers at admission. Systolic blood pressure, diastolic blood pressure, and mean blood pressure of enrolled patients were also measured, and the means of them were $144.78 \pm 19.62$, $90.27 \pm 15.67$, and $108.44 \pm 16.60 \mathrm{mmHg}$, respectively.

In terms of laboratory characteristics, the essential object in this study was the serum soluble klotho level. On the ELISA assay, the medium level of serum soluble klotho was $447.13 \pm 156.07 \mathrm{pg} / \mathrm{mL}$. Enrolled patients had normal hemoglobin and albumin levels, which were $120.33 \pm 12.04$ and $38.01 \pm 6.64 \mathrm{~g} / \mathrm{L}$, respectively. The level of cholesterol was at a normal level $(3.96 \pm 0.73 \mathrm{mmol} / \mathrm{L})$. Due to existing primary diseases, the creatinine level of enrolled patients was high, the mean of which was $842.88 \pm 150.92 \mu \mathrm{mol} / \mathrm{L}$. Some patients have slight inflammation at admission, revealed by a slightly elevated level of C-reactive protein $(8.26 \pm 5.68 \mathrm{mg} / \mathrm{L})$. Considering that klotho plays a vital role in the regulation of calcium and phosphorus metabolism, the level of serum, calcium, and phosphorus is measured as well, $2.45 \pm 0.20$ and $1.79 \pm 0.24 \mathrm{mmol} / \mathrm{L}$, respectively.

CIMT and AAC grade was introduced into this study to verify the cardiovascular function of enrolled patients. Representative ultrasonography images of CIMT 
Table 1 Demographic, clinical and laboratory characteristics of enrolled patients

\begin{tabular}{|c|c|c|c|c|}
\hline Variables & Total & Klotho > medium & Klotho $<$ medium & $P$ value \\
\hline Age & $60.13 \pm 9.30$ & $58.64 \pm 9.67$ & $60.84 \pm 9.10$ & 0.244 \\
\hline \multicolumn{5}{|l|}{ Gender } \\
\hline Male & $65(58.04 \%)$ & $22(61.11 \%)$ & $43(56.58 \%)$ & 0.650 \\
\hline Body mass index & $22.75 \pm 3.07$ & $22.67 \pm 3.47$ & $22.80 \pm 2.88$ & 0.832 \\
\hline Dialysis duration (months) & $63.09 \pm 29.54$ & $61.75 \pm 29.10$ & $63.72 \pm 29.92$ & 0.743 \\
\hline Hypertension & $84(75.00 \%)$ & $28(77.78 \%)$ & $56(73.68 \%)$ & 0.640 \\
\hline Diabetes mellitus & $25(22.32 \%)$ & $8(22.22 \%)$ & $17(22.37 \%)$ & 0.986 \\
\hline Diastolic blood pressure $(\mathrm{mmHg})$ & $90.27 \pm 15.67$ & $93.06 \pm 14.94$ & $88.95 \pm 15.92$ & 0.232 \\
\hline Mean blood pressure $(\mathrm{mmHg})$ & $108.44 \pm 16.60$ & $111.39 \pm 16.39$ & $107.04 \pm 16.62$ & 0.197 \\
\hline Serum soluble Klotho (pg/mL) & $447.13 \pm 156.07$ & $645.58 \pm 115.03$ & $353.13 \pm 44.91$ & $<0.001$ \\
\hline Hemoglobin (g/L) & $120.33 \pm 12.04$ & $118.83 \pm 11.44$ & $121.04 \pm 12.32$ & 0.367 \\
\hline Albumin (g/L) & $38.01 \pm 6.64$ & $39.50 \pm 5.64$ & $37.30 \pm 6.99$ & 0.102 \\
\hline Cholesterol (mmol/L) & $3.96 \pm 0.73$ & $4.11 \pm 0.73$ & $3.88 \pm 0.72$ & 0.132 \\
\hline Creatinine $(\mu \mathrm{mol} / \mathrm{L})$ & $842.88 \pm 150.92$ & $849.78 \pm 169.32$ & $839.61 \pm 142.47$ & 0.741 \\
\hline $\mathrm{C}$ reactive protein $(\mathrm{mg} / \mathrm{L})$ & $8.26 \pm 5.68$ & $8.43 \pm 5.83$ & $8.18 \pm 5.65$ & 0.828 \\
\hline
\end{tabular}

CIMT, carotid intima-media thickness; AAC, abdominal aorta calcification.

measurement were listed in Figure 2. As shown in Table 1, most patients have cardiovascular dysfunction in varying degrees. The medium level of CIMT was $0.92 \pm 0.23 \mathrm{~mm}$, and the mean grade of the AAC level was $3.96 \pm 3.82$ points.

\section{Klotho participates in cardiovascular dysfunction}

All enrolled patients were then divided into two groups according to whether their klotho levels were more than the median level or not. It was found there was no significant difference between them, expecting two cardiovascular parameters compared to the baseline characteristics in the two groups. Patients with lower levels of serum soluble klotho have a much higher level of CIMT than those with lower levels $(1.03 \pm 0.22$ vs. $0.85 \pm 0.20 \mathrm{~mm}, \mathrm{P}<0.001)$. Similarly, patients with lower klotho levels had higher levels of AAC grade $(5.04 \pm 3.93$ vs. $1.69 \pm 2.30$ points, $\mathrm{P}<0.001)$.

Univariate and multivariate linear regression analysis was further performed to determine the relationship between serum soluble klotho level and cardiovascular dysfunction, as shown in Table 2. The results showed that risk factors of cardiovascular dysfunction (both high CIMT and AAC grade) were smoking history and lower level of serum soluble klotho. 

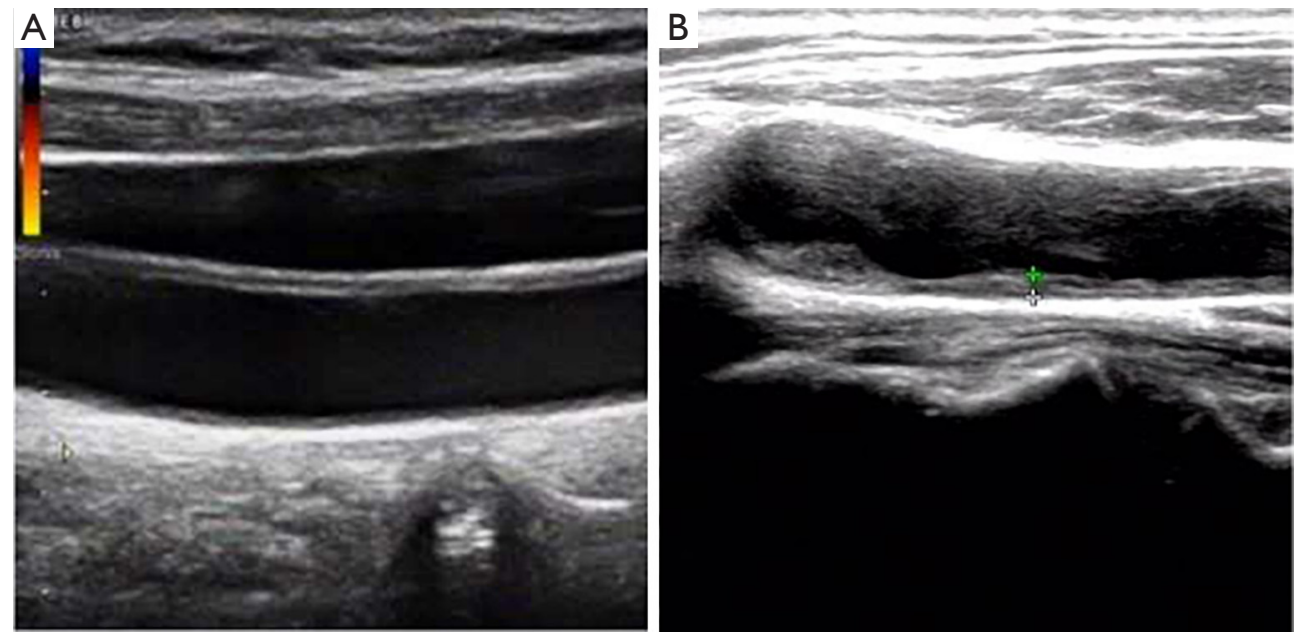

Figure 2 Two representative ultrasonography images of CIMT measurement. (A) Normal carotid artery, (B) thickened carotid artery.

Table 2 Univariate and multivariate linear regression analysis of cardiovascular dysfunction in hemodialysis patients

\begin{tabular}{|c|c|c|c|c|}
\hline Variables & \multicolumn{2}{|c|}{ Univariate analysis } & \multicolumn{2}{|c|}{ Multivariate analysis } \\
\hline \multicolumn{5}{|l|}{ CIMT } \\
\hline Current or former smokers & 0.006 & $0.118(0.034$ to 0.201$)$ & 0.014 & $0.099(0.020$ to 0.178$)$ \\
\hline Serum soluble Klotho & $<0.001$ & $-0.184(-0.270$ to -0.099$)$ & $<0.001$ & $-0.172(-0.257$ to -0.088$)$ \\
\hline Current or former smokers & 0.003 & 2.117 (0.735 to 3.499$)$ & 0.007 & 1.785 (0.503 to 3.067$)$ \\
\hline Serum soluble Klotho & $<0.001$ & $-3.345(-4.747$ to -1.943$)$ & $<0.001$ & $-3.128(-4.499$ to -1.758$)$ \\
\hline
\end{tabular}

CIMT, carotid intima-media thickness; AAC, abdominal aorta calcification.

\section{Effects of tan on cardiovascular events and mortality of patients}

Some enrolled patients were given sodium tan sulfonate injection as a role of a cardiovascular protector according to their willingness. Finally, 71 patients were given tan, and 41 patients refused. All of them were followed up for at least 12 months. The changes of serum soluble klotho and cardiovascular parameters were collected 12 months later, and endpoint events were also recorded during the followup. As shown in Table 3, 7 of 71 patients $(9.86 \%)$ who were given tan had cardiovascular events, among whom 4 patients $(5.63 \%)$ died. In total, 10 patients $(14.08 \%)$ who were given $\tan$ died due to all causes. However, 11 of 41 patients $(26.83 \%)$ not given tan had cardiovascular events, among whom 5 patients (12.20\%) died. Seven patients not given tan died of all causes.
It was revealed that given tan helped MHD patients to significantly improve the level of serum soluble klotho $(490.23 \pm 153.97 \mathrm{pg} / \mathrm{mL}$ after using tan versus $444.49 \pm 143.32 \mathrm{pg} / \mathrm{mL}$ before using tan, $\mathrm{P}=0.042$ ) comparing the changes of serum soluble klotho and cardiovascular parameters in two groups. Also, CIMT increased from $0.94 \pm 0.22$ to $0.98 \pm 0.26 \mathrm{~mm}$ in patients given tan during follow-up while it increased from $0.92 \pm 0.21$ to $0.98 \pm 0.25 \mathrm{~mm}$ in patients not given tan. Similarly, AAC grade increased from $3.36 \pm 3.40$ to $3.76 \pm 3.86$ points and from $2.94 \pm 2.66$ to $3.85 \pm 3.11$ points in patients given and not given tan, respectively. It could be found that the rate of cardiovascular thickening and calcification in patients given tan was slower than that in patients not given tan, though no significant difference was found due to the small sample size.

Kaplan-Meier curves of freedom for the three endpoint events are shown in Figure 3. The results proved that all 
Table 3 Comparison of baseline and follow-up data of patients receiving or not receiving Tan

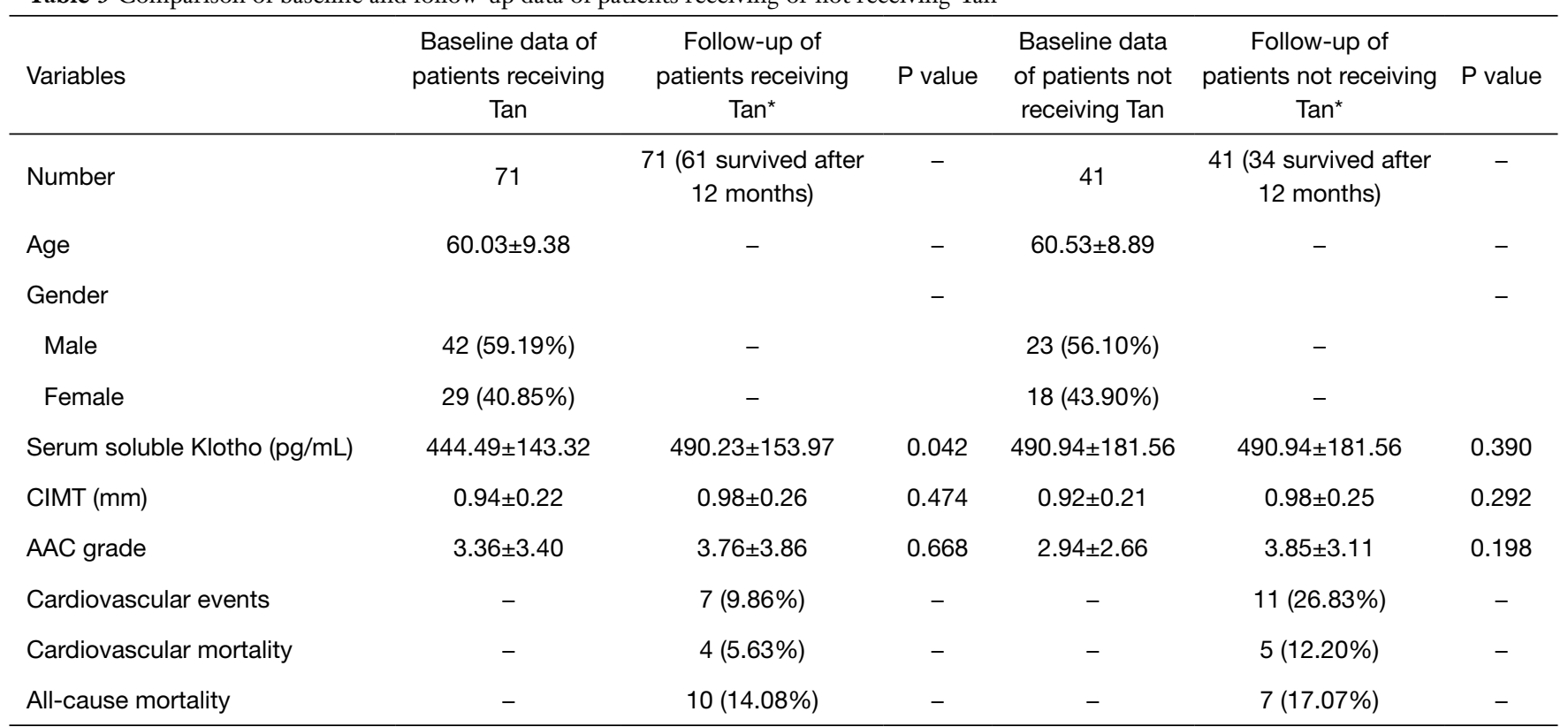

*, these data were collected 12 months later.
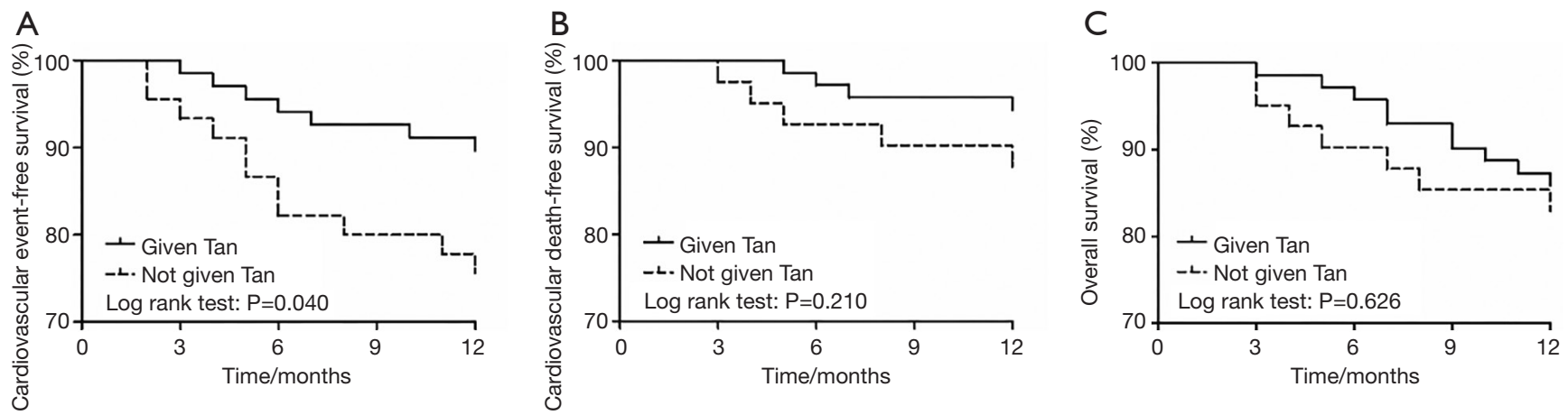

Figure 3 Kaplan-Meier survival curves of the endpoint events: (A) cardiovascular events, (B) cardiovascular mortality and (C) all-cause mortality.

event-free survivals were higher in the patient groups who were given $\tan$ than those not given tan. However, only cardiovascular event-free survival was significantly higher in patients given tan according to the log-rank test $(\mathrm{P}=0.040)$.

\section{Discussion}

Here we performed a prospective before-after study in MHD patients, trying to address two clinical questions. One is the role of serum soluble klotho in cardiovascular dysfunction, and the other is the effect of $\tan$ on cardiovascular events. The results showed that klotho played a protective role in
MHD patients, and decreased levels of serum soluble klotho contributed to cardiovascular dysfunction. Tan was found to be beneficial for the prevention of cardiovascular events for the first time, as we know, which may partially owe to the increase of klotho levels.

Klotho is secreted mainly by the kidney, and it has been revealed that the level of serum soluble klotho was decreased in the early stages of CKD due to persistent chronic renal injury (21). The most important physiological function of klotho is to be involved in the regulation of aging and metabolism of calcium and phosphorus as a cofactor for fibroblast growth factor 23 (FGF23) (20). It also 
plays a vital role in the pathogenesis of hypertension by reducing peroxide-induced endothelial cell apoptosis and improving the bioactivity of vascular endothelial cells (22). However, no significant relationship between klotho level and hypertension could be found in this study, which may be because almost all enrolled patients had hypertension (75.00\%), and small sample size limited the further analysis.

Cardiovascular thickening and calcification are some of the most common complications in MHD patients, and their persistent development dramatically increases the incidence of CVD (15). CIMT and AAC grade were used in this study to judge the development of cardiovascular thickening and calcification. The results observed the rising of both CIMT and AAC grades in patients with a lower level of klotho, which were like previous studies (13,15,23-25). The mechanism of how decreased serum soluble klotho level leads to cardiovascular thickening and calcification is not clear to date. The potential explanation is that the decrease of klotho results in the rising of phosphorus and further create calcium deposits in vascular endothelium (26). Also, recent studies have revealed that klotho exerted some direct effects on vascular smooth muscle cells (VSMC). For example, klotho was found to maintain VSMC integrity by interacting with transient receptor potential channel-1 and vascular endothelial growth factor receptor-2 (27). In vitro experiments showed as well, klotho suppressed tumor necrosis factor $\alpha$ induced activation of adhesion molecules and inflammatory signaling pathways (28).

Some other traditional risk factors were previously reported to be related to the incidence of cardiovascular thickening and calcification, like age, weight, and smoking history (25). This study also verified that smoking history functioned as a risk factor for cardiovascular thickening and calcification. Nevertheless, age and weight showed no significant relationship with them.

After given tan, the level of serum soluble klotho was significantly increased in MHD patients. To date, no related studies were performed to investigate the relationship between tan and klotho proteins. However, tan has been reported to improve kidney injury in chronic kidney disease, which may explain the reason tan improved the klotho level in this study. It has been reported tan attenuated renal fibrosis and inflammation through inhibiting transforming growth factor $\beta$ and NF-kappaB signaling pathways and reducing oxidative stress in rat models $(29,30)$. Additionally, tan was reported to inhibit renal epithelial cell fibrosis through the epithelial-myofibroblast transdifferentiation pathway (31). The improvement of renal function may contribute to the increase of serum klotho levels in this study. Furthermore, increased klotho slows down the thickening and calcification of blood vessels.

Besides, tan has functioned as a protector of CVD for hundreds of years. $\mathrm{Li}$ et al. have found that $\tan$ can inhibit VSMC proliferation through the miR-145/CD40 signaling pathway (32). Furthermore, tan has also been reported to prevent the proliferation of basilar artery smooth muscle cells in hypertension rats through 3'-phosphoinositidedependent kinase/AKT signaling pathway (33). Therefore, the results in this study showed an improvement in cardiovascular event-free survival in patients given tan compared with those not given tan as expected.

Cardiovascular mortality and all-cause mortality are the other two main endpoint events in this study. Unfortunately, no significant effects of $\tan$ on them were observed. On the one hand, the small sample size of this study limited further analysis. Nevertheless, the therapeutic effect of heart disease depends on the timing and method of treatment.

Several limitations of this study must be highlighted. First, this is a non-randomized study. Using tan is on the willingness of patients, which may generate some bias in this study. Second, the sample size of our study is not comprehensive enough to answer all relevant questions, including the relationship between tan and measured cardiovascular parameters or cardiovascular mortality. Third, some upstream and downstream targets or related proteins of klotho protein are not measured, like FGF23 and parathyroid hormone. Their measurements may supply a clearer explanation for this study. Thus, more welldesigned trials are still needed.

In conclusion, this prospective before-after study preliminarily enrolling 112 MHD patients verifies the vital role of serum soluble klotho in cardiovascular dysfunction of MHD patients. Besides, this study indicates that tan can effectively increase the level of serum soluble klotho and further reduce the incidence of cardiovascular events in MHD patients. The effects of $\tan$ on cardiovascular dysfunction and mortality of MHD patients are still to be explored.

\section{Acknowledgments}

Funding: The work was supported by the "Six One" Project by the Jiangsu Commission of Health (No. LGY201703).

\section{Footnote}

Reporting Checklist: The authors have completed the 
STROBE reporting checklist. Available at http://dx.doi. org/10.21037/apm-20-1172

Data Sharing Statement: Available at http://dx.doi. org/10.21037/apm-20-1172

Conflicts of Interest: All authors have completed the ICMJE uniform disclosure form (available at http://dx.doi. org/10.21037/apm-20-1172). The authors have no conflicts of interest to declare.

Ethical Statement: The authors are accountable for all aspects of the work in ensuring that questions related to the accuracy or integrity of any part of the work are appropriately investigated and resolved. The study was performed in compliance with the ethical principles of the Declaration of Helsinki (as revised in 2013) and had been approved by the ethics committee of Taixing People's Hospital (approval number: TC2017011). All adult patients had signed the informed consent.

Open Access Statement: This is an Open Access article distributed in accordance with the Creative Commons Attribution-NonCommercial-NoDerivs 4.0 International License (CC BY-NC-ND 4.0), which permits the noncommercial replication and distribution of the article with the strict proviso that no changes or edits are made and the original work is properly cited (including links to both the formal publication through the relevant DOI and the license). See: https://creativecommons.org/licenses/by-nc-nd/4.0/.

\section{References}

1. Ni LH, Yuan C, Song KY, et al. Efficacy and safety of cinacalcet and active vitamin $\mathrm{D}$ in the treatment of secondary hyperparathyroidism in patients with chronic kidney disease: a network meta-analysis. Ann Transl Med 2019;7:322.

2. Tan J. Forty-billion medical expenditure for dialysis patients in China. Kidney Int 2019;96:523.

3. Zhou L, Zeng XX, Fu P. Community Hemodialysis in China: Opportunities and Challenges. Chin Med J (Engl) 2017;130:2143-6.

4. Roberts MA, Pilmore HL, Tonkin AM, et al. Challenges in blood pressure measurement in patients treated with maintenance hemodialysis. Am J Kidney Dis 2012;60:463-72.

5. Georgianos PI, Sarafidis PA, Lasaridis AN. Arterial stiffness: a novel cardiovascular risk factor in kidney disease patients. Curr Vasc Pharmacol 2015;13:229-38.

6. Saran R, Li Y, Robinson B, et al. US Renal Data System 2015 Annual Data Report: Epidemiology of Kidney Disease in the United States. Am J Kidney Dis 2016;67:Svii, S1-305.

7. Sarafidis PA, Loutradis C, Karpetas A, et al. Ambulatory Pulse Wave Velocity Is a Stronger Predictor of Cardiovascular Events and All-Cause Mortality Than Office and Ambulatory Blood Pressure in Hemodialysis Patients. Hypertension 2017;70:148-57.

8. Georgianos PI, Georgianos PI, Sarafidis PA, et al. Adverse effects of conventional thrice-weekly hemodialysis: is it time to avoid 3-day interdialytic intervals? Am J Nephrol 2015;41:400-8.

9. Kuro-o M, Matsumura Y, Aizawa H, et al. Mutation of the mouse klotho gene leads to a syndrome resembling ageing. Nature 1997;390:45-51.

10. Chen TH, Kuro-O M, Chen CH, et al. The secreted Klotho protein restores phosphate retention and suppresses accelerated aging in Klotho mutant mice. Eur J Pharmacol 2013;698:67-73.

11. Kurosu H, Yamamoto M, Clark JD, et al. Suppression of aging in mice by the hormone Klotho. Science 2005;309:1829-33.

12. Kuro-O M. The Klotho proteins in health and disease. Nat Rev Nephrol 2019;15:27-44.

13. Memmos E, Sarafidis P, Pateinakis P, et al. Soluble Klotho is associated with mortality and cardiovascular events in hemodialysis. BMC Nephrol 2019;20:217.

14. Abdallah E, Mosbah O, Khalifa G, et al. Assessment of the relationship between serum soluble Klotho and carotid intima-media thickness and left ventricular dysfunction in hemodialysis patients. Kidney Res Clin Pract 2016;35:42-9.

15. Zheng S, Zheng Y, Jin L, et al. Relationship between Serum Soluble Klotho Protein and Coronary Artery Calcification and Prognosis in Patients on Maintenance Hemodialysis. Iran J Public Health 2018;47:510-8.

16. Gao J, Yang G, Pi R, et al. Tanshinone IIA protects neonatal rat cardiomyocytes from adriamycin-induced apoptosis. Transl Res 2008;151:79-87.

17. Wang J, Dong MQ, Liu ML, et al. Tanshinone IIA modulates pulmonary vascular response to agonist and hypoxia primarily via inhibiting $\mathrm{Ca} 2+$ influx and release in normal and hypoxic pulmonary hypertension rats. Eur J Pharmacol 2010;640:129-38.

18. Stumpf C, Fan Q, Hintermann C, et al. Anti-inflammatory 
effects of danshen on human vascular endothelial cells in culture. Am J Chin Med 2013;41:1065-77.

19. Jia LQ, Yang GL, Ren L, et al. Tanshinone IIA reduces apoptosis induced by hydrogen peroxide in the human endothelium-derived EA.hy926 cells. J Ethnopharmacol 2012;143:100-8.

20. Cai H, Lu R, Zhang M, et al. Serum Soluble Klotho Level Is Associated with Abdominal Aortic Calcification in Patients on Maintenance Hemodialysis. Blood Purif 2015;40:120-6.

21. Rotondi S, Pasquali M, Tartaglione L, et al. Soluble alpha -Klotho Serum Levels in Chronic Kidney Disease. Int J Endocrinol 2015;2015:872193.

22. Gao D, Zuo Z, Tian J, et al. Activation of SIRT1 Attenuates Klotho Deficiency-Induced Arterial Stiffness and Hypertension by Enhancing AMP-Activated Protein Kinase Activity. Hypertension 2016;68:1191-9.

23. Yu L, Kang L, Ren XZ, et al. Circulating alpha-Klotho Levels in Hemodialysis Patients and Their Relationship to Atherosclerosis. Kidney Blood Press Res 2018;43:1174-82.

24. Milovanova LY, Dobrosmyslov IA, Milovanov YS, et al. Fibroblast growth factor-23 (FGF-23)/soluble Klotho protein (sKlotho)/sclerostin glycoprotein ratio disturbance is a novel risk factor for cardiovascular complications in ESRD patients receiving treatment with regular hemodialysis or hemodiafiltration. Ter Arkh 2018;90:48-54.

25. Marçais C, Maucort-Boulch D, Drai J, et al. Circulating Klotho Associates With Cardiovascular Morbidity and Mortality During Hemodialysis. J Clin Endocrinol Metab 2017;102:3154-61.

26. John GB, Cheng CY, Kuro-o M. Role of Klotho in aging, phosphate metabolism, and CKD. Am J Kidney Dis

Cite this article as: $\mathrm{Xu} Q$, Li H, Zhang $\mathrm{X}$, Ding $\mathrm{H}$, Cao J. Tanshinone IIA elevates serum soluble klotho levels and decreases cardiovascular events in patients on maintenance hemodialysis: a prospective before-after study. Ann Palliat Med 2020;9(4):2131-2140. doi: 10.21037/apm-20-1172
2011;58:127-34.

27. Kusaba T, Okigaki M, Matui A, et al. Klotho is associated with VEGF receptor-2 and the transient receptor potential canonical-1 $\mathrm{Ca} 2+$ channel to maintain endothelial integrity. Proc Natl Acad Sci U S A 2010;107:19308-13.

28. Maekawa Y, Ishikawa K, Yasuda O, et al. Klotho suppresses TNF-alpha-induced expression of adhesion molecules in the endothelium and attenuates NF-kappaB activation. Endocrine 2009;35:341-6.

29. Wang DT, Huang RH, Cheng X, et al. Tanshinone IIA attenuates renal fibrosis and inflammation via altering expression of TGF-beta/Smad and NF-kappaB signaling pathway in 5/6 nephrectomized rats. Int Immunopharmacol 2015;26:4-12.

30. Chen X, Wu R, Kong Y, et al. Tanshinone IIA attenuates renal damage in STZ-induced diabetic rats via inhibiting oxidative stress and inflammation. Oncotarget 2017;8:31915-22.

31. Cao L, Huang B, Fu X, et al. Effects of tanshinone IIA on the regulation of renal proximal tubular fibrosis. Mol Med Rep 2017;15:4247-52.

32. Li Y, Chen F, Guo R, et al. Tanshinone A inhibits homocysteine-induced proliferation of vascular smooth muscle cells via miR-145/CD40 signaling. Biochem Biophys Res Commun 2020;522:157-63.

33. Yu ZL, Wang JN, Wu XH, et al. Tanshinone IIA Prevents Rat Basilar Artery Smooth Muscle Cells Proliferation by Inactivation of PDK1 During the Development of Hypertension. J Cardiovasc Pharmacol Ther 2015;20:563-71.

(English Language Editor: J. Chapnick) 